\title{
Grounding the data. A response to: Population finiteness is not a concern for null hypothesis significance testing when studying human behavior
}

\author{
Thomas V. Pollet* \\ Department of Experimental and Applied Psychology, VU University Amsterdam, Amsterdam, Netherlands
}

Keywords: cross-cultural research, $p$-value, statistical inference, evolutionary psychology, ecological fallacy

\section{A commentary on}

Population finiteness is not a concern for null hypothesis significance testing when studying

OPEN ACCESS

Edited by:

Martin Fieder,

University of Vienna, Austria

Reviewed by:

Rosemary Hopcroft,

University of North Carolina at

Charlotte, USA

Georg Primes,

University of Vienna, Austria

*Correspondence:

Thomas V. Pollet,

t.v.pollet@vu.n

Specialty section: This article was submitted to Evolutionary Psychology and Neuroscience,

a section of the journal

Frontiers in Psychology

Received: 25 June 2015

Accepted: 24 July 2015

Published: 06 August 2015

Citation:

Pollet TV (2015) Grounding the data. A response to: Population finiteness is

not a concern for null hypothesis

significance testing when studying human behavior.

Front. Psychol. 6:1169. doi: 10.3389/fpsyg.2015.01169 human behavior. A reply to Pollet (2013)

by Quillien, T. (2015). Front. Neurosci. 9:81. doi: 10.3389/fnins.2015.00081

I am thankful to Quillien (2015) for his response to my paper (Pollet, 2013), as it allows clarifying my position. Firstly, I would like to underline that the purpose of my paper was to flesh out the (implicit) statistical assumptions underpinning cross-cultural correlations. However, what I highlighted is but a side-issue when working with macro-level cross-cultural data (e.g., Poortinga, 1989; Mace and Pagel, 1994; Pollet et al., 2014). I would like to bring the discussion back to "earth" and clarify why I believe, in contrast to Quillien (2015), that finite populations might be problematic in this context.

I put forward that the sampling units for macro-level cross-cultural correlations are finite. In contrast, Quillien argues that this does not have to be the case. I believe for the examples I cited (Pollet, 2013: Table 1), the statistical data are clearly scores derived countries, states, etc. and are therefore by their (implicit) definition finite. Data at country/state/region level are very much unlike a population of people (Kuppens and Pollet, 2014) or an experiment where we can gather new, independent observations. Quillien presents no argument that these observed entities such as states/countries/etc. are in fact infinite but rather argues that the scores these researchers use represent something else.

Let us return to a specific example we both discussed, U.S. state scores (e.g., Kanazawa, 2006; Eppig et al., 2011). I put forward that if we sampled all possible units, here: all U.S. state scores, then there is no probability for the statistical population of U.S. states (for that point in time). The sample (U.S. States) matches the population we wish to make statistical inferences about (U.S. states). If the observations we sample are from a finite population (U.S. states), this needs to be corrected for (Pollet, 2013). Once we have sampled the last U.S. state, the population pool is empty: we sampled and measured everything. Not explicitly defining the "population" does not alter this, nor does assuming that these state scores are part of a larger, potentially infinite, whole. Many authors, such as Quillien, might want to make statistical or logical inferences beyond these scores, for example to other macro-level units or the "human mind," but what we have in terms of data are U.S. state scores, plain and simple. These are by their very nature finite (fixed number of U.S. states). Quillien's argument thus seems to crucially rest on the claim that the observed unit of analysis is 
not a U.S. state score but rather something else. What this different unit of analysis would be is typically not clearly defined, neither by Quillien nor by the authors cited in Pollet (2013: Table 1). For now, like Quillien, let us assume the unit of analysis is some (aggregate) human social unit relevant to some evolutionary process. Such a stance, i.e., the data representing something else than a U.S. state score, is in my view deeply problematic. Firstly, if one assumes the scores are something else, then one needs to explicitly define the unit of analysis $a$ priori, otherwise it seems reasonable that the unit of analysis is indeed a U.S. state score. Let us tentatively define this alternative unit of analysis as "a social unit in which humans live(d) relevant to an evolutionary process." Why would a U.S. state then be representative of the pool of such social units in which humans lived? Perhaps these data can indeed tell us something about U.S. states, but it is unclear whether any documented statistical relationship would hold for other units fitting the broader definition. It is unclear whether any statistical inference can be done beyond U.S. states, as that is all we have. There is no logical reason to assume that any statistical relationship found for U.S. states should hold, for other "human social units," such as for example: world regions, Canadian provinces, Polynesian chiefdoms, 19th century German states, hunter gatherer populations,.... Let alone that these different "human social units" can be meaningfully lumped together and assumed to be governed by the same evolutionary process, as Quillien seems to imply. In addition, suppose that we do follow Quillien's logic and pretend the pool is larger, and even infinite, then a different problem still arises: we have clearly drawn a biased sample (Good and Hardin, 2012). "Traditional" statistical inference based on rejecting a null hypothesis in the population cannot be applied in the first place as there was no random sampling. For example, Canadian provinces are

\section{References}

Eppig, C., Fincher, C. L., and Thornhill, R. (2011). Parasite prevalence and the distribution of intelligence among the states of the USA. Intelligence 39, 155-160. doi: 10.1016/j.intell.2011. 02.008

Freedman, D. A. (1999). Ecological inference and the ecological fallacy. Int. Encycl. Soc. Behav. Sci. 6, 4027-4030.

Good, P. I., and Hardin, J. W. (2012). Common Errors in Statistics (and How to Avoid Them). Hoboken, NJ: John Wiley \& Sons.

Kanazawa, S. (2006). IQ and the wealth of states. Intelligence 34, 593-600. doi: 10.1016/j.intell.2006.04.003

Kuppens, T., and Pollet, T. V. (2014). Mind the level: problems with two recent nation-level analyses in psychology. Front. Psychol. 5:1110. doi: $10.3389 /$ fpsyg. 2014.01110

Mace, R., and Pagel, M. (1994). The comparative method in anthropology. Curr. Anthropol. 35, 549-564. doi: 10.1086/204317

Pollet, T. V. (2013). Much ado about p. What does a $\mathrm{p}$ value mean when testing hypotheses with aggregated cross-cultural data in the field of evolution and human behavior? Front. Psychol. 4:734. doi: 10.3389/fpsyg.2013. 00734 underrepresented, absent even, in our sample, while they are in our statistical population.

Perhaps I am thus opposed to logical induction, and view moving from U.S. states to other not clearly defined "units" as problematic and Quillien does not. I will leave the reader to decide but it seems a much safer bet to stick to inferences about U.S. states, and not even rely on these data to make any inference on, for example, Canadian provinces. Ideally, researchers would then define and measure those, rather than assuming that one process must govern all these units.

Finally, if we take these state level data to be representative of processes at an individual level ("the human mind"), as several authors including Quillien seem to suggest, then the ecological fallacy looms: inferences from one level of statistical analysis need not correspond to a different level of analysis (see Robinson, 1950; Freedman, 1999; Kuppens and Pollet, 2014; Pollet et al., 2014). These arguments have been made at length elsewhere and will not be reiterated here.

In summary, in my view, the statistical inferences we can make based on units such as U.S. state scores can be about nothing else but U.S. states, as this is the only unit being sampled. One might want to strengthen the statistical and logical inference based on those data but this is likely invalid: either due to sampling bias and/or the ecological fallacy. I therefore maintain that the $p$-values commonly used for statistical inference are inappropriate for macro-level cross-cultural correlations when the sample matches the population closely.

\section{Acknowledgments}

I am thankful to Gert Stulp, Toon Kuppens and Peter Dekker for helpful discussions on this topic. Any mistakes are obviously my own.

Pollet, T. V., Tybur, J. M., Frankenhuis, W. E., and Rickard, I. J. (2014). What can cross-cultural correlations teach us about human nature? Hum. Nat. 25, 410-429. doi: 10.1007/s12110-014-9206-3

Poortinga, Y. H. (1989). Equivalence of cross-cultural data: an overview of basic issues. Int. J. Psychol. 24, 737-756. doi: 10.1080/00207598908247842

Quillien, T. (2015). Population finiteness is not a concern for null hypothesis significance testing when studying human behavior. A reply to Pollet (2013). Front. Neurosci. 9:81. doi: 10.3389/fnins.2015.00081

Robinson, W. S. (1950). Ecological correlations and the behavior of individuals. Am. Sociol. Rev. 15, 351-357.

Conflict of Interest Statement: The author declares that the research was conducted in the absence of any commercial or financial relationships that could be construed as a potential conflict of interest.

Copyright (c) 2015 Pollet. This is an open-access article distributed under the terms of the Creative Commons Attribution License (CC BY). The use, distribution or reproduction in other forums is permitted, provided the original author (s) or licensor are credited and that the original publication in this journal is cited, in accordance with accepted academic practice. No use, distribution or reproduction is permitted which does not comply with these terms. 